\title{
ON A METHOD FOR OBTAINING APPROXIMATE SOLUTIONS TO STURM-LIOUVILLE PROBLEMS
}

\author{
SERGE ABRATE
}

\begin{abstract}
A method presented recently to find approximate solutions to a Sturm-Liouville problem is shown to be a special case of a classical weighted residual method. The choice of approximation functions and convergence of the method are also examined.
\end{abstract}

\section{INTRODUCTION}

In a recent article [1], Chambers presented a method for obtaining an approximate solution to the Sturm-Liouville problem defined by the differential equation

$$
-\frac{d}{d x}\left(p(x) \frac{d u}{d x}\right)+q(x) u=f(x)
$$

for $x_{1} \leq x \leq x_{2}$, and the boundary conditions

$$
-p\left(x_{1}\right) u^{\prime}\left(x_{1}\right)+r_{1} u\left(x_{1}\right)=g_{1}, \quad p\left(x_{2}\right) u^{\prime}\left(x_{2}\right)+r_{2} u\left(x_{2}\right)=g_{2} .
$$

Integrating the differential equation (1.1) from $x_{1}$ to $x_{2}$, one obtains the "consistency equation"

$$
\int_{x_{1}}^{x_{2}} q u d x+r_{1} u\left(x_{1}\right)+r_{2} u\left(x_{2}\right)=\int_{x_{1}}^{x_{2}} f d x+g_{1}+g_{2}
$$

and one seeks an approximate solution of the form

$$
w=\alpha+\beta x+\gamma x^{2} .
$$

The constants $\alpha, \beta$ and $\gamma$ are selected so that $w$ satisfies the boundary conditions (equation (1.2)) and the consistency equation (1.3).

The purpose of the present note is to show that this method is simply a particular case of a well-known variational approximation method for which convergence has been established and error analyses are available.

\section{WeIGHTED RESIDUAL METHOD}

Following the standard approach [2], multiplying the differential equation (1.1) by a test function $\eta$ and integrating from $x_{1}$ to $x_{2}$, gives

$$
\left[-\eta(x) p(x) u^{\prime}(x)\right]_{x_{1}}^{x_{2}}+\int_{x_{1}}^{x_{2}} \eta^{\prime} p u^{\prime} d x+\int_{x_{1}}^{x_{2}} \eta q u d x=\int_{x_{1}}^{x_{2}} \eta f d x .
$$

Received by the editor May 3, 1994 and, in revised form, November 7, 1994.

1991 Mathematics Subject Classification. Primary 34B24, 65L60. 
Using the boundary conditions (1.2), we find

$$
\begin{aligned}
\eta\left(x_{1}\right) r_{1} u\left(x_{1}\right)- & \eta\left(x_{1}\right) g_{1}+\eta\left(x_{2}\right) r_{2} u\left(x_{2}\right)-\eta\left(x_{2}\right) g_{2} \\
& +\int_{x_{1}}^{x_{2}} \eta^{\prime}(x) p u^{\prime} d x+\int_{x_{1}}^{x_{2}} \eta q u d x=\int_{x_{1}}^{x_{2}} \eta f d x .
\end{aligned}
$$

Equation (2.2) is the weak formulation of the problem defined by equations (1.1-2). An $N$-term approximate solution is sought in the form

$$
u_{N}=\sum_{j=1}^{N} c_{j} \phi_{j}(x),
$$

where the $c_{j}$ 's are constants to be determined. With the Petrov-Galerkin weighted residual method [2], $N$ linearly independent test functions $\eta_{i}$ may be taken to be different from the approximation functions $\phi_{j}$. A system of $N$ linear algebraic equations is obtained after substituting equation (2.3) into equation (2.2). The unknown constants $c_{j}$ are determined by solving the system of linear algebraic equations

$$
\left[k_{i j}\right]\left\{c_{j}\right\}=\left\{F_{i}\right\},
$$

where

$$
\begin{gathered}
k_{i j}=\int_{x_{1}}^{x_{2}}\left[\eta_{i}^{\prime} p \phi_{j}^{\prime}+\eta_{i} q \phi_{j}\right] d x+\eta_{i}\left(x_{2}\right) r_{2} \phi_{j}\left(x_{2}\right)+\eta_{i}\left(x_{1}\right) r_{1} \phi_{j}\left(x_{j}\right), \\
F_{i}=\eta_{i}\left(x_{2}\right) g_{2}+\eta_{i}\left(x_{1}\right) g_{1}+\int_{x_{1}}^{x_{2}} \eta_{i} f d x .
\end{gathered}
$$

When the test functions are the same as the approximation functions, the method is called the Bubnov-Galerkin method.

The method presented by Chambers [1] can be seen as a one-term PetrovGalerkin approximation with a polynomial approximation function and $\eta=1$ since, after substituting $\eta=1$ into equation (2.2), the consistency equation (1.3) is recovered. The approximation function used satisfied both boundary conditions of the problem.

In the following, we show that: (1) better results are obtained using more terms in the approximation (equation (2.3)); (2) the approximation functions need not satisfy the boundary conditions; (3) faster convergence is obtained when each approximation function satisfies the boundary conditions of the problem.

\section{EXAMPLE}

For the example treated in [1],

$$
p=q=r_{2}=f=x_{2}=1, \quad x_{1}=r_{1}=g_{1}=g_{2}=0,
$$

the boundary conditions become

$$
u^{\prime}(0)=0, \quad u(1)+u^{\prime}(1)=0
$$

and the exact solution is given by

$$
u=1-e^{-1} \cosh (x) .
$$


The test functions are taken as

$$
\eta_{i}=x^{i-1}, \quad i=1,2, \ldots, N .
$$

Three different approximations are obtained from different choices of the approximation functions.

Approximation I. First, consider polynomial approximation functions that satisfy the boundary conditions of the problem:

$$
\phi_{1}=x^{2}-3
$$

and, for $i=2$ to $N$,

$$
\phi_{i}=x^{i}-\frac{i+1}{i+2} x^{i+1} .
$$

Substituting equations $(3.1,3.4-6)$ into equations $(2.4-5)$, we can determine approximations of any order. For a three-term approximation,

$$
\left[\begin{array}{ccc}
-\frac{14}{3} & \frac{19}{48} & \frac{29}{100} \\
-\frac{9}{4} & \frac{3}{5} & \frac{7}{15} \\
-\frac{22}{15} & \frac{8}{15} & \frac{248}{525}
\end{array}\right] \quad\left\{\begin{array}{l}
a_{1} \\
a_{2} \\
a_{3}
\end{array}\right\}=\left\{\begin{array}{l}
1 \\
\frac{1}{2} \\
\frac{1}{3}
\end{array}\right\}
$$

From equation (3.6), the one-, two- and three-term approximations are obtained as

$$
\begin{gathered}
u_{1}=-\frac{3}{14}\left(x^{2}-3\right) \\
u_{2}=-\frac{386}{1833}\left(x^{2}-3\right)+\frac{80}{1833}\left(x^{2}-\frac{3}{4} x^{3}\right) \\
u_{3}=-0.210707\left(x^{2}-3\right)+2.60750 \times 10^{-2}\left(x^{2}-\frac{3}{4} x^{3}\right) \\
+2.19926 \times 10^{-2}\left(x^{3}-\frac{4}{5} x^{4}\right) .
\end{gathered}
$$

Equation (3.7) was also obtained in [1]. Results from equations (3.8-10) are compared with the exact solution in Table 1 for $x=0,1 / 2,1$. As the number of terms increases, the approximate solution rapidly converges to the exact solution. Therefore, the method used can produce very accurate solutions.

Approximation II. Because of the boundary condition $u^{\prime}(0)=0$ and the fact that the differential equation remains unchanged when $x$ is changed to $-x$, there is a strong indication that the solution is an even function. Of course, this is confirmed by the exact solution (equation (3.3)). Therefore, the approximation functions are taken to be polynomials with even powers of $x$. That is

$$
\phi_{i}=x^{2 i-2}-\frac{2 i-1}{2 i+1} x^{2 i}
$$

for $i \geq 1$. Results in Table 1 indicate a slightly faster convergence with this set of approximation functions. When available, special insight into the solution of the problem can be used in the selection of the approximation functions to speed up convergence, but is not needed. 
TABLE 1. Comparison of approximate and exact solutions

Solution $\begin{gathered}\text { Number } \\ \text { of terms }\end{gathered} \quad x=0 \quad x=1 / 2 \quad x=1$

$\begin{array}{llll}\text { Exact } & 0.6321206 & .5851696 & .4323324\end{array}$

$\begin{array}{lllll}\text { Approx. I } & 1 & 0.6428571 & 0.5892857 & 0.4285714 \\ & 2 & 0.6317512 & 0.5859247 & 0.4320786 \\ & 3 & 0.6321217 & 0.5851687 & 0.4323320\end{array}$

$\begin{array}{lllll}\text { Approx. II } & 1 & 0.6428571 & 0.5892857 & 0.4285714 \\ & 2 & 0.6320960 & 0.5852211 & 0.4323144 \\ & 3 & 0.6321206 & 0.5851695 & 0.4323324\end{array}$

$\begin{array}{lllll}\text { Approx. III } & 1 & 0.5 & 0.5 & 0.5 \\ & 2 & 0.6551724 & 0.5517241 & 0.4482759 \\ & 3 & 0.6319520 & 0.5853326 & 0.4323882 \\ & 4 & 0.6321407 & 0.5853898 & 0.4323286 \\ & 5 & 0.6321209 & 0.5851780 & 0.4323324\end{array}$

Approximation III. In this case, no attempt is made to select approximate functions that satisfy the boundary conditions of the problem. Taking

$$
\phi_{i}=x^{i-1}, \quad i=1, \ldots, N,
$$

we find that the approximation converges to the exact solution (Table 1). It was also verified that the boundary conditions (equation (3.2)) are also very well approximated as $N \geq 3$. The results show that with Approximation III, convergence is slower than with Approximations I and II.

Remark. Equation (1.1) can be written as

$$
A u=f,
$$

where the operator $A$ is defined as

$$
A=-\frac{d}{d x}\left(p(x) \frac{d}{d x}\right)+q(x) .
$$

Using the results obtained in equation (2.2), we find the inner products (3.15)

$$
\begin{aligned}
(A u, \eta)= & \int_{x_{1}}^{x_{2}} \eta \cdot A u d x=\eta\left(x_{1}\right) r_{1} u\left(x_{1}\right)-\eta\left(x_{1}\right) g_{1}+\eta\left(x_{2}\right) r_{2} u\left(x_{2}\right)-\eta\left(x_{2}\right) g_{2} \\
& +\int_{x_{1}}^{x_{2}} \eta^{\prime}(x) p u^{\prime} d x+\int_{x_{1}}^{x_{2}} \eta q u d x
\end{aligned}
$$


and

$$
(f, \eta)=\int_{x_{1}}^{x_{2}} \eta f d x .
$$

Then, equation (2.2) can be written as

$$
(A u, \eta)=(f, \eta) \text {. }
$$

The operator $A$ is symmetric $((A u, \eta)=(u, A \eta))$ if $g_{1}=g_{2}=0$, and then it is also positive $((A u, u)>0)$. Therefore, according to the minimum functional theorem [2], the solution to the strong problem defined by equations $(1.1,2)$ corresponds to the minimum of the quadratic functional

$$
\begin{aligned}
Q(u)= & (A u, u)-2(f, u)=\eta\left(x_{1}\right) r_{1} u\left(x_{1}\right)-\eta\left(x_{1}\right) g_{1}+\eta\left(x_{2}\right) r_{2} u\left(x_{2}\right)-\eta\left(x_{2}\right) g_{2} \\
& +\int_{x_{1}}^{x_{2}} \eta^{\prime}(x) p u^{\prime} d x+\int_{x_{1}}^{x_{2}} \eta q u d x-2 \int_{x_{1}}^{x_{2}} u f d x .
\end{aligned}
$$

Using the Rayleigh-Ritz method, one seeks an $N$-term approximation of the form given by equation (2.3), and the minimum of the functional $Q$ is reached when the $N$ equations

$$
\sum_{j=1}^{N}\left(A \phi_{j}, \phi_{i}\right) c_{j}-\left(f, \phi_{i}\right)=0
$$

are satisfied. The weighted residual method adopted here is applicable to the most general Sturm-Liouville problem, whereas a quadratic functional $Q$ can only be found when $g_{1}=g_{2}=0$. However, when such a functional can be found, as with the present example, the Rayleigh-Ritz method can be used. It can be shown that approximation III above leads to the same set of equations as the Rayleigh-Ritz approach using the approximation functions given by equations (3.12).

\section{Conclusions}

In this article, a general weighted residual approach for Sturm-Liouville problems was presented. A method proposed recently by Chambers is shown to be a special case of the classical Petrov-Galerkin method. In addition, we show that the Petrov-Galerkin approach quickly converges to the exact solution even when approximation functions do not each satisfy the boundary conditions of the problem. However, faster convergence is obtained when each approximation function does not satisfy the boundary conditions. The Petrov-Galerkin approach does not require the existence of a quadratic functional to be minimized and can be used to solve all Sturm-Liouville problems. Quadratic functionals are shown to exist only for a restricted class of Sturm-Liouville problems.

\section{BIBLIOGRAPHY}

1. Ll. G. Chambers, A quick way of obtaining an approximate solution to a Sturm-Liouville problem, Math. Comp. 62 (1994), 577-579.

2. J. N. Reddy, Applied functional analysis and variational methods in engineering, McGrawHill, New York, 1986.

Department of Mechanical and Aerospace Engineering and Engineering Mechanics, UNiversity of MisSouri-Rolla, Rolla, Missouri 65401 